$\mathrm{E}$

EVALUAR
2019, Vol. 19, No. 1

ISSN 1667-4545

Recuperado de https://revistas.unc.edu.ar/index.php/revaluar

Laboratorio de Evaluación Psicológica y Educativa Facultad de Psicología - Universidad Nacional de Córdoba

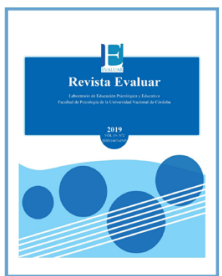

\title{
Actitud sobre el ejercicio físico y los deportes: Un estudio psicométrico en estudiantes universitarios
}

\author{
Attitudes towards physical exercise and sports: \\ A psychometric study in college students
}

\author{
Andrés Cruz-Santos ${ }^{* 1}$, Juan A. González-Rivera ${ }^{2}$, Adam Rosario-Rodríguez ${ }^{1}$ \\ 1 - Universidad Carlos Albizu, San Juan, Puerto Rico. \\ 2 - Ponce Health Sciences University, San Juan University Center, Puerto Rico.
}
Introducción
Método
Resultados
Discusión
Conclusión
Referencias

Recibido: 29/01/2019 Revisado: 06/03/2019 Aceptado: 31/03/2019

\section{Resumen}

El presente estudio analiza las propiedades psicométricas de la Escala de Actitud Hacia el Ejercicio Físico y los Deportes (AHEFD-11) en una muestra de estudiantes adultos puertorriqueños. Un total de 255 puertorriqueños participaron en este estudio de carácter exploratorio y psicométrico. Los resultados reflejaron una solución de tres factores como la más apropiada para explicar la varianza en las puntuaciones. Estos factores fueron identificados como actitud hacia el ejercicio físico, pasión por el deporte y disposición. Un total de 11 reactivos cumplieron con los criterios de discriminación y obtuvieron cargas factoriales apropiadas. El índice de consistencia interna alfa de Cronbach de la escala fue de .88. Además, se encontró una correlación positiva y estadísticamente significativa entre la actitud hacia el ejercicio físico y el deporte, el bienestar psicológico y la satisfacción con la vida $(r=.27, p<.001)$. AHEFD-11 permitirá nuevas investigaciones sobre el fenómeno del ejercicio y el deporte en Puerto Rico.

Palabras clave: actividad fisica, propiedades psicométricas, validación, ejercicio, deportes

\begin{abstract}
The following study analyses the psychometric properties of the Attitudes Towards Physical Exercise and Sport Scale (AHEFD-11) in a sample of Puerto Rican adults. A total of 255 Puerto Ricans participated in this exploratory and psychometric study. The results reflected a three-factor solution as the most appropriate to explain the variance in the scores. These factors were identified as attitude to physical exercise, passion for sport and disposition. A total of 11 items met the discrimination criteria and obtained appropriate factor loadings. The reliability index (Cronbach's alpha) of the scale was .88 . In addition, a positive and statistically significant correlation was found between attitude towards physical exercise and sports, psychological well-being and life satisfaction $(r=.27, p<.001)$. The AHEFD-11 will allow new research on the phenomenon of physical exercise and sports in Puerto Rico.
\end{abstract}

Keywords: physical activity, psychometric properties, validation, exercise, sports

\footnotetext{
* Correspondencia a: Andrés Cruz Santos; Po Box 834 Cidra P.R 00739. Tel.: 787- 436-5950. Email: andcruz@albizu.edu

Cómo citar este artículo: Cruz-Santos, A., González-Rivera, J. A., \& Rosario-Rodríguez, A. (2019). Actitud sobre el ejercicio físico y los deportes: Un estudio psicométrico en estudiantes universitarios. Revista Evaluar, 19(2), 58-72. Recuperado de https://revistas.unc.edu.ar/index.php/revaluar
} 


\section{Introducción}

En las últimas décadas, se ha experimentado un aumento en la producción de nuevas tecnologías que facilitan las responsabilidades y quehaceres cotidianos. Cada día se observa cómo estos avances tecnológicos llegan al mercado para de alguna manera servir como extensión del cuerpo humano, ayudando a las personas a llevar a cabo actividades de manera simple y con menos esfuerzo físico. No obstante, estos avances han hecho que las personas disminuyan la actividad física durante sus vidas, aún si reconocen que el ejercicio físico está vinculado con beneficios psicológicos (Hassmen, Koivula, \& Uutela, 2000).

La Organización Mundial de la Salud (World Health Organization [WHO], 2010) expresa que la inactividad física es un problema de salud pública a nivel mundial. Esto provoca un sinnúmero de enfermedades que han afectado a la población de manera negativa en estos últimos años. La causa principal del sobrepeso es la inactividad física y el desequilibrio energético entre las calorías consumidas y las gastadas. Al menos un $60 \%$ de la población mundial no realiza la actividad física necesaria para obtener beneficios para la salud (WHO, 2010). También, la WHO (2010) expone como consecuencia de la inactividad física a enfermedades como: cardiopatías coronarias, accidentes cardiovasculares, diabetes, hipertensión, distintos tipos de cáncer, como el cáncer del colon y de mama, y trastornos mentales como la depresión. El ejercicio físico habitual proporciona múltiples beneficios para la salud del individuo que lo realiza, dentro de estos, disminuir las probabilidades de mortalidad por causa de enfermedades crónicas cardiovasculares, metabólicas y oncológicas (Rebolledo-Cobos, Teixeira, \& Correa, 2014). Meseguer-Zafra et al. (2019) mencionan que en la actualidad se están implementando diferentes programas de ejercicio físico como alternativa de tratamiento. De esta manera, la actividad física se presenta como un instrumento útil para reducir la mortalidad derivada de este tipo de enfermedades $\mathrm{y}$, por tanto, se debe fomentar como parte activa en el tratamiento.

Por una parte, epidemiólogos han estudiado los comportamientos de evasión de la actividad física como parte activa en el tratamiento y han determinado que estos impactan negativamente en la salud (Pedišić, Dumuid, \& Olds, 2017). En efecto, existe una inquietud sobre si las personas que no realizan actividad física pueden gozar de bienestar psicológico y estar satisfechos con la vida. A estos efectos, esta investigación fue dirigida al desarrollo y validación una escala para medir la actitud hacia el ejercicio físico y el deporte y, a su vez, auscultar si existe una correlación significativa entre el ejercicio, el bienestar psicológico y la satisfacción con la vida en una muestra de estudiantes universitarios puertorriqueños.

\section{Ejercicio Físico}

Barbany (2015) explica que el acto del ejercicio físico está compuesto por la acción de componentes de tipo motor: los músculos de tipo esquelético forman parte del proceso por el cual la energía química se convierte directamente en energía mecánica, lo que genera una fuerza que permite que los movimientos se lleven a cabo. Otra definición expone que el ejercicio físico regular, el cual hace referencia al esfuerzo físico prolongado, representa una medida directa del estado general de salud biológica, especialmente del sistema cardiovascular (Taylor, Buskirk, \& Henschel, 1955). En cambio, en el ámbito de la psicología de la actividad física y el deporte, se lo ha vinculado en los últimos años con un concepto llamado fortaleza mental, definido como 
"un conjunto de valores, actitudes, emociones y cogniciones que influyen en la manera como una persona accede, responde y evalúa eventos de alto nivel de exigencia para lograr sus metas de manera consistente" (Gucciardi, Gordon, \& Dimmock, 2009). También se menciona que el ejercicio físico permite a las personas llevar a cabo actividades cotidianas sin fatigarse (Malina \& Katzmarzyk, 2006). A diferencia del ejercicio físico, la actividad física como caminar más rápido y aumentar la intensidad del trabajo, puede ayudar a cambiar el estilo de vida (Haith-Cooper, Waskett, Montague, \& Horne, 2018). Según el American College of Sports Medicine (2000), los términos ejercicio físico y actividad física sostienen una similitud importante entre ellos. Sin embargo, también presentan diferencias. El ejercicio físico corresponde a un acto planificado y organizado de actividad física, mientras que la actividad física solo define el movimiento corporal que se puede llevar a cabo de distintas maneras (Hassmén et al., 2000). Otra diferencia entre el ejercicio físico y la actividad física es que la actividad física es un estado de condición que le permite a un individuo realizar actividades cotidianas sin sufrir fatiga y disfrutar adecuadamente de sus objetivos diarios. En este sentido, la actividad física se observa de manera histórica en tres componentes: fuerza muscular y resistencia, resistencia cardiorrespiratoria y habilidad motora.

La actividad física, a diferencia del ejercicio físico, no tiene que ser programada y organizada (Latorre-Román et al., 2017). Del mismo modo, el término bienestar físico está compuesto por varias características como aptitud respiratoria y composición corporal, que incluye a su vez distribución de grasa, fuerza muscular, flexibilidad y resistencia (Pollock et al., 1998). No obstante, Malina y Katzmarzyk (2006) explican la actividad física como una conducta con distintas dimensiones que conlleva el movimiento del cuerpo a través del espacio. La actividad física se observa a menudo como un gasto de energía y estrés asociada a la pérdida de peso y la sensación de bienestar. Además, se observa como un componente específico de las destrezas de la aptitud física. El contexto sociocultural también define el tipo de actividad física que se lleva a cabo, como por ejemplo deportes, juego, educación, trabajo y ejercicio, entre otros. Estas actividades están fuertemente influenciadas por la cultura (Latorre-Román et al., 2017). Una buena forma de reconocer que se está realizando una actividad física adecuada es cuando el ritmo cardíaco aumenta, pero todavía se puede hablar (WHO, 2010).

En un estudio llevado a cabo en Inglaterra, Escocia, Gales e Irlanda del Norte se presentan algunas reglas mínimas sobre el volumen, la duración, la frecuencia y el tipo de actividad física necesarios durante toda la vida con el fin de lograr beneficios generales para el bienestar. Este estudio promueve también la actividad física, el deporte, el ejercicio físico, y los métodos de transportación activos como el caminar para lograr un bienestar adecuado (Department of Health, Physical Activity, Health Improvement and Protection, 2011). Además, Franco et al. (2015) mencionan unas estrategias para aumentar la práctica de ejercicio físico entre las personas que incluyen: 1 ) Aumentar la conciencia de los beneficios y minimizar los riesgos percibidos de la actividad física. En ocasiones, las personas desconocen los beneficios que puede brindar el ejercicio físico. 2) Mejorar el acceso ambiental y económico a las oportunidades del ejercicio físico. La accesibilidad de un lugar donde se puedan llevar a cabo ejercicios a bajo costo es mínima en el occidente (WHO, 2010). Jiménez, Martínez, Miró y Sánchez (2008) argumentan que la prescripción de ejercicio físico orientado a obtener determinados beneficios para la salud se relaciona frecuentemente con diferentes parámetros (frecuencia, duración, intensidad, 
tipo de ejercicio, etc.). Por otra parte, Hassmén et al. (2000) comparten algunas recomendaciones para incluir el ejercicio físico en la vida de las personas y promover y mantener un buen estado de salud. Los adultos de 18 a 65 años necesitan 30 minutos de actividad física por al menos cinco días a la semana o 20 minutos de actividad física de alta intensidad al menos tres días a la semana. La combinación de ejercicio moderado y de alta intensidad puede ser utilizada para alcanzar el mínimo recomendado de actividad física por semana.

Pollock et al. (1998) definen los requisitos que se deben cumplir para lograr una capacidad cardiorrespiratoria y una composición corporal adecuada mediante el ejercicio físico, como, por ejemplo, la frecuencia y la planificación del entrenamiento: este debe ser llevado a cabo por un mínimo de tres días semanales y un máximo de cinco días semanales. La intensidad del entrenamiento debe ser de un 55/65\% - 90\% del ritmo cardíaco máximo de cada persona (HRmax), o $40 / 50 \%$ - 85\% del consumo de reserva de oxígeno (V.O2R). La duración del entrenamiento debe ser de 20 a 60 minutos de actividad aeróbica continua o intermitente. En cuanto al modo o la actividad, se recomienda cualquier ejercicio que use grupos grandes de músculos, que se puedan trabajar continuamente como caminar-excursionismo, correr-trotar, ciclismo-montar la bicicleta, baile, remar, subir escaleras, nadar, patinar y varios deportes de alto rendimiento (Pollock et al., 1998). Por otro lado, el término actitud procede de la psicología social. Son muchas las definiciones que se le han dado a lo largo del siglo XX desde que nació el término como un constructo para explicar y medir el grado por el que las personas tienden a comportarse de manera selectivamente distinta en situaciones similares. La variación de una a otra definición es producto de la orientación que se le dé a la misma. De esta manera, algu- nos autores dan prioridad a elementos cognitivos, otros a elementos afectivos y otros a elementos comportamentales (Dosil-Díaz, 2002).

Instrumentos para evaluar la actitud hacia el ejercicio físico y el deporte

La mayoría de los instrumentos con que se cuenta en nuestro ámbito han sido desarrollados en países sudamericanos y no toman en consideración la relación con el bienestar psicológico y la satisfacción con la vida. En los últimos años, los estudios sobre actitudes y la actividad física y el deporte han aumentado considerablemente (Dosil-Díaz, 2002). Los trabajos que más relevancia han tenido se deben a Kenyon (1968) quien desarrolló una línea de investigación sobre el tema que se cristalizó con la construcción de la Escala de Actitudes hacia la Actividad Física (ATPA). De manera análoga, uno de los instrumentos utilizados en investigaciones en Sudamérica es la Escala de Actitudes hacia la Actividad Física y el Deporte (Dosil-Díaz, 2002). Esta escala pretende ser un instrumento de medida que valora la actitud de cualquier sujeto hacia la actividad física y el deporte, independientemente de si es o no practicante. Dosil-Díaz (2002) recomienda que próximas investigaciones profundicen en el constructo actitud, teniendo en cuenta la relación, en principio positiva, entre la práctica y la actitud, pero sin asumir que se tiene una actitud negativa hacia el deporte solo porque no se practica ejercicio. A estos efectos, esta investigación tiene como objetivo el desarrollo y la validación de una escala que mida la actitud hacia el ejercicio físico $\mathrm{y}$ el deporte $\mathrm{y}$, a su vez, examinar la relación de estos con la satisfacción con la vida y el bienestar psicológico y auscultar si existen diferencias estadísticamente significativas entre las medias de actitud hacia el ejercicio físico y el deporte entre 
hombres y mujeres. Los investigadores establecieron como hipótesis que existen relaciones significativas entre la actitud hacia el ejercicio físico y los deportes y la satisfacción con la vida y el bienestar psicológico. Además, que existen diferencias significativas en cuanto a la actitud hacia el ejercicio físico y los deportes en relación con el género.

\section{Método}

Diseño de Investigación

El diseño que se utilizó en esta investigación fue ex post facto, de tipo instrumental (Montero \& León, 2007).

\section{Participantes}

El procedimiento para la selección de los participantes fue por disponibilidad, de tipo no probabilístico. La muestra estuvo compuesta por 255 estudiantes universitarios adultos reclutados a través de correo electrónico y redes sociales. En la Tabla 1 se presentan los datos sociodemográficos de los participantes. Para participar de este estudio, se establecieron los siguientes criterios de inclusión: (1) ser mayor de 21 años de edad, (2) tener la capacidad de leer en español, (3) ser puertorriqueños, (4) ser estudiantes activos de una universidad.

\section{Instrumentos}

Cuestionario de Datos Generales. Desarrollamos un cuestionario ad-hoc de datos sociodemográficos para obtener información relacionada con la edad, sexo, estado civil, preparación académica, ingresos anuales, frecuencia de actividad física, entre otras variables que permitieron la descripción de la muestra del estudio.

Tabla 1

Información sociodemográfica de la muestra.

\begin{tabular}{lcr}
\hline \multicolumn{1}{c}{ Variables } & $\boldsymbol{f}$ & $\mathbf{\%}$ \\
\hline Edad & & \\
21 - 30 & 159 & 62.4 \\
31 - 40 & 62 & 24.3 \\
42 - 50 & 25 & 9.8 \\
51 - 65 & 9 & 3.5 \\
Sexo & & \\
Femenino & 203 & 79.6 \\
Masculino & 52 & 20.4 \\
Preparación Académica & & \\
Escuela superior o menos & 10 & 3.9 \\
Bachillerato & 96 & 37.6 \\
Maestría & 109 & 42.7 \\
Doctorado & 40 & 15.7 \\
Estado civil & & \\
Casado/Convivencia & 79 & 31.0 \\
Soltero & 162 & 63.5 \\
Divorciado & 12 & 4.7 \\
Otros & 2 & 0.8 \\
Ingresos Anuales & & \\
\$0 - \$25,000 & 169 & 66.3 \\
\$26,000 - \$50,000 & 55 & 21.6 \\
\$51,000 - \$100,00 & 22 & 8.6 \\
\$101,000 o más & 9 & 3.5 \\
Frecuencia de actividad física & & \\
Diariamente & 33 & 12.9 \\
Tres veces por semana & 75 & 29.9 \\
Dos veces por semana & 35 & 13.7 \\
Una vez por semana & 33 & 12.9 \\
\hline & & \\
& &
\end{tabular}

Nota. $N=255$.

Escala de Actitudes Hacia el Ejercicio Físico y el Deporte (AHEFD-11). Gracias a los trabajos de Dosil-Díaz (2002) y la construcción de la Escala de Actitudes hacia la Actividad Física y el Deporte en Sudamérica. A pesar de que los términos son parecidos, a diferencia de la escala de Dosil-Díaz (2002), que solo trabaja el concepto de actividad física, la escala AHEFD-11 también aborda conceptos más centrados en el ejercicio físico. A pesar de que los conceptos actividad 
física y ejercicio físico son similares, existen diferencias significativas: a diferencia del ejercicio físico, que conlleva una planificación (Pollock et al., 1998), la actividad física no necesariamente es planificada o estructurada. Los autores de esta investigación desarrollaron 13 reactivos que se agruparon en tres subescalas: actitud hacia el ejercicio, pasión por el deporte y disposición. Estas dimensiones integran las actitudes, pasión y práctica de actividad física y deporte que puede tener un individuo. El análisis factorial exploratorio confirmó la presencia de estas tres dimensiones. En cuanto a las subescalas del instrumento, la dimensión denominada actitud hacia el ejercicio está asociada a la actitud que poseen las personas en cuanto a sus prioridades, consideraciones, tiempo y dedicación que muestran cuando realizan algún ejercicio físico. Estas características son importantes para el resultado de una actividad física adecuada (Pollock et al., 1998).

La escala fue desarrollada por el autor principal para medir la actitud hacia la actividad física y el deporte desde una perspectiva multidimensional. La versión original de la escala estaba constituida por 13 reactivos. La creación de los reactivos fue inspirada por los estudios de Pollock et al. (1998) y la WHO (2010). Estos reactivos fueron agrupados dentro de una estructura de tres factores según la taxonomía del creador de la escala: actitud hacia el ejercicio físico, pasión por el deporte y disposición. Se utilizó un formato de cuatro respuestas estilo Likert que fluctuaban desde Totalmente en desacuerdo hasta Totalmente en acuerdo en continuo numérico del 1 al 4. El puntaje más bajo que se podía obtener en la versión original es 13 y el más alto es 52 , donde una mayor puntuación indica una actitud más positiva hacia el ejercicio físico.

Escala Breve de Bienestar Psicológico. Este instrumento fue desarrollado por Ryff (1989) y adaptado a la población puertorriqueña por González-Rivera, Quintero-Jiménez, Veray-Alicea y Rosario-Rodríguez (2016). Se compone de 17 reactivos organizados en una escala tipo Likert de seis puntos que va de Fuertemente en desacuerdo a De acuerdo (por ejemplo; No tengo claro lo que quiero conseguir en la vida). La escala consta de cuatro factores: 1) dominio del entorno, 2) propósito de vida, 3) auto-aceptación, y 4) autonomía, y tiene un índice de confiabilidad alfa de Cronbach de .85 .

Escala de Satisfacción con la Vida. Esta escala es la versión en español del instrumento Satisfaction With Life Scale (SWLS) de Diener, Emmons, Larsen y Griffin (1985). Estos autores y la WHO (2010) consideran que la satisfacción con la vida constituye el componente cognitivo del bienestar subjetivo. El instrumento está constituido por un total de 5 reactivos organizados en una escala tipo Likert de siete puntos que va de Muy en desacuerdo a Muy de acuerdo (por ejemplo, Soy bastante bueno manejando las muchas responsabilidades de mi vida cotidiana; Estoy satisfecho con mi vida; Si pudiera vivir mi vida de nuevo, no cambiaría nada). Este instrumento tiene un índice de consistencia interna alfa de Cronbach de .88 .

\section{Procedimientos Generales}

Los datos fueron recopilados de forma electrónica durante el mes de diciembre 2018. Se distribuyó un anuncio a través de redes sociales (Facebook) y correos electrónicos para que las personas interesadas pudieran participar de la investigación. Los participantes tenían autorización para compartir el anuncio en sus redes sociales y por correo electrónico, con el fin de ampliar la muestra mediante el efecto "bola de nieve". Para la versión electrónica, se utilizó la plataforma de 
PsychData (s. f.). Este es un sitio web dirigido a crear instrumentos en línea para el campo de la psicología que permitió que el instrumento fuera colocado en internet con todas las especificaciones del Comité de Ética en la Investigación de la Universidad Carlos Albizu recinto de San Juan.

Una vez recopilados los datos, analizamos los mismos utilizando el sistema para análisis estadísticos Statistical Package for the Social Sciences (IBM Corporation, 2016). Específicamente, realizamos análisis descriptivos de la muestra, análisis de reactivos para conocer su índice de discriminación, análisis de factores utilizando el método de extracción máxima verosimilitud con rotación oblicua, análisis de confiabilidad para calcular el coeficiente alfa de Cronbach y división en mitades de Spearman-Brown, análisis de comparación y análisis de correlación. Todos los procedimientos de este estudio fueron aprobados por el Comité Institucional de Revisión (IRB por sus siglas en inglés) de la Universidad Carlos Albizu de San Juan, Puerto Rico.

\section{Resultados}

Análisis factorial exploratorio

Para determinar la validez de construcción lógica del instrumento, llevamos a cabo un análisis factorial utilizando el método de extracción de máxima verosimilitud con rotación oblicua para factores correlacionados, identificando aquellos factores que expliquen el 5\% o más de la varianza, como sugiere Hatcher (1994). Como criterios de aceptación, consideramos aquellos reactivos con una carga factorial mayor a .50 en un solo factor (Stevens, 2002). Los resultados mostraron una estructura de tres factores que explicaban el $69 \%$ de la varianza de los datos originales. Sin embargo, cuando revisamos las cargas factoriales de cada reactivo, se identificaron dos ítems que no cumplían con los criterios de aceptación detallados anteriormente y que fueron eliminados de los análisis posteriores: (3) Alguna vez me he acostado o levantado antes de mi horario acostumbrado para poder llevar a cabo un ejercicio físico o deportivo, y (10) Si dejo de hacer ejercicio físico o deporte por una semana, observo o experimento una reducción en mi condición fisi$c a$. Luego, procedimos a realizar nuevamente el análisis factorial con los 11 reactivos restantes.

En esta segunda ocasión, los resultados mostraron nuevamente una estructura de tres factores que explicaban el $69 \%$ de la varianza, del cual el $46 \%$ es explicado por el Factor 1, el 13\% es explicado por el Factor 2 y el $10 \%$ es explicado por el Factor 3. Al revisar las cargas factoriales de estos reactivos, observamos que todos cumplieron los criterios de inclusión. Las pruebas de Kaiser-Meyer-Olkin (KMO = .890) y la prueba de esfericidad de Bartlett $\left(\chi_{(55)}^{2}=1297.078, p<\right.$ .001 confirmaron la adecuación de los datos de muestreo para el análisis. En la Tabla 2 se presentan las cargas factoriales obtenidas por los 11 reactivos, cuya distribución por dimensión fue la siguiente: 5 reactivos en el primer factor, 3 en el segundo y 3 en el tercero.

Al revisar detenidamente la agrupación de los reactivos por su carga factorial, se determinó que los reactivos $11,1,13,7$ y 8 pertenecen a la dimensión de la actitud de la persona hacia el ejercicio físico. Los reactivos 6,12 y 4 pertenecen a la dimensión de la pasión por el deporte que muestran las personas. Finalmente, los reactivos 5,9 y 2 pertenecen a la dimensión de la predisposición de la persona para realizar actividad física.

\section{Análisis de reactivos}

Se analizó la discriminación de los 11 ítems de la AHEFD-11 en sus respectivos factores y en 
Tabla 2

Reactivos cuyas cargas factoriales fueron superiores a $.50 \mathrm{y}$ sus factores correspondientes.

\begin{tabular}{|c|c|c|c|c|}
\hline & Reactivo & 1 & 2 & 3 \\
\hline 11. & Entre mis prioridades en la vida está el ejercicio físico y deportivo. & .890 & & \\
\hline 1. & El ejercicio físico y deportivo ocupa un lugar importante en mi vida. & .872 & & \\
\hline 13. & $\begin{array}{l}\text { En general, me considero una persona que tiene una actitud favorable o de buena predispo- } \\
\text { sición hacia el ejercicio físico o deportivo. }\end{array}$ & 685 & & \\
\hline 7. & $\begin{array}{l}\text { Le dedico más tiempo a la práctica de algún ejercicio físico o deportivo que a pasar tiempo } \\
\text { con mis amistades. }\end{array}$ & .587 & & \\
\hline 8. & $\begin{array}{l}\text { Mis deseos de realizar o practicar ejercicios físicos no disminuyen aún cuando no pueda } \\
\text { practicarlos durante una semana completa. }\end{array}$ & .559 & & \\
\hline 6. & $\begin{array}{l}\text { Alguna vez me he acostado o levantado antes de mi horario acostumbrado para poder ver } \\
\text { una retransmisión deportiva. }\end{array}$ & & .702 & \\
\hline 12. & Mis programas favoritos son los deportivos. & & 684 & \\
\hline 4. & $\begin{array}{l}\text { Siempre que tengo la oportunidad asisto a manifestaciones o espectáculos deportivos pre- } \\
\text { sencialmente (juegos de pelota, baloncesto, voleibol, entre otras). }\end{array}$ & & .628 & \\
\hline 5. & $\begin{array}{l}\text { Me gustaría tener siempre un tiempo en la semana para dedicarlo al ejercicio físico o } \\
\text { deporte. }\end{array}$ & & & .756 \\
\hline 9. & $\begin{array}{l}\text { Mis deseos de realizar o practicar ejercicio físico aumentan cuando veo a una persona } \\
\text { practicándolo. }\end{array}$ & & & 649 \\
\hline 2. & $\begin{array}{l}\text { Si tuviera una tarde libre, emplearía una parte de mi tiempo en realizar algún ejercicio } \\
\text { físico o deportivo. }\end{array}$ & & & .639 \\
\hline
\end{tabular}

Nota. Factor 1 = Actitud hacia el ejercicio físico; Factor 2 = Pasión por el deporte; Factor 3 = Disposición.

la escala total mediante el índice de correlación ítem-total. Los índices de discriminación de los ítems para la dimensión actitud hacia el ejercicio físico fluctuaron entre .56 y .81 , mientras que para la pasión deportiva oscilaron entre .56 y .57. Asimismo, la puntuación del factor disposición osciló entre .57 y .64 (véase Tabla 3). Todos los índices de discriminación de la versión trifactorial de la AHEFD-11 sobrepasaron el valor mínimo recomendado de .30 (Field, 2013).

\section{Análisis de confiabilidad}

Los 11 reactivos fueron sometidos a un análisis de consistencia interna para determinar el índice de confiabilidad de la escala. Para ello utilizamos dos métodos: el coeficiente alfa de Cronbach y la división en mitades de Spearman-Brown. Los resultados mostraron un coeficiente alfa de .72 y de .90 para la prueba Spearman-Brown. Utilizamos estos mismos análisis para cada una de las dimensiones identificadas en el análisis de factores. En la Tabla 4 se presentan el coeficiente alfa de Cronbach, el coeficiente de la prueba Spearman-Brown, la media y la desviación estándar de la versión final de la AHEFD-11 y sus subescalas.

\section{Análisis de correlación}

Se realizó un análisis correlacional lineal de los tres factores identificados y la AHEFD-11, la escala de bienestar psicológico y la escala de satisfacción con la vida utilizando el coeficiente producto-momento de Pearson. Los resultados se muestran en la Tabla 4. Como se puede observar, todos los coeficientes $r$ de Pearson obtenidos en los análisis fueron estadísticamente significativos. 
Tabla 3

Índices de discriminación y varianza explicada de los ítems en sus tres dimensiones (actitud hacia el ejercicio físico, pasión por el deporte y disposición).

\begin{tabular}{|c|c|c|c|c|c|}
\hline & Ítems & $r_{\text {bis }}^{1}$ & $r_{\text {bis }}^{2}$ & $r_{\text {bis }}{ }^{3}$ & $r_{\text {bis }}{ }^{\mathrm{T}}$ \\
\hline 11. & Entre mis prioridades en la vida está el ejercicio físico y deportivo. & .81 & & & .75 \\
\hline 1. & El ejercicio físico y deportivo ocupa un lugar importante en mi vida. & .81 & & & .74 \\
\hline 13. & $\begin{array}{l}\text { En general, me considero una persona que tiene una actitud favorable o de buena predis- } \\
\text { posición hacia el ejercicio físico o deportivo. }\end{array}$ & .73 & & & .72 \\
\hline 7. & $\begin{array}{l}\text { Le dedico más tiempo a la práctica de algún ejercicio físico o deportivo que a pasar tiem- } \\
\text { po con mis amistades. }\end{array}$ & .56 & & & .53 \\
\hline 8. & $\begin{array}{l}\text { Mis deseos de realizar o practicar ejercicio físico no disminuyen, aún cuando no pueda } \\
\text { practicarlos durante una semana completa. }\end{array}$ & .63 & & & .62 \\
\hline 6. & $\begin{array}{l}\text { Alguna vez me he acostado o levantado antes de mi horario acostumbrado para poder ver } \\
\text { una retransmisión deportiva. }\end{array}$ & & .56 & & .43 \\
\hline 12. & Mis programas favoritos son los deportivos. & & .56 & & .51 \\
\hline 4. & $\begin{array}{l}\text { Siempre que tengo la oportunidad, asisto a manifestaciones o espectáculos deportivos } \\
\text { presencialmente (juegos de pelota, baloncesto, voleibol, entre otros). }\end{array}$ & & .56 & & .46 \\
\hline 5. & $\begin{array}{l}\text { Me gustaría tener siempre un tiempo en la semana para dedicarlo al ejercicio físico o } \\
\text { deporte. }\end{array}$ & & & .64 & .52 \\
\hline 9. & $\begin{array}{l}\text { Mis deseos de realizar o practicar ejercicio físico aumentan cuando veo a una persona } \\
\text { practicándolo. }\end{array}$ & & & .57 & .47 \\
\hline 2. & $\begin{array}{l}\text { Si tuviera una tarde libre, emplearía una parte de mi tiempo en realizar algún ejercicio } \\
\text { físico o deportivo. }\end{array}$ & & & .64 & .62 \\
\hline
\end{tabular}

Nota. $r_{\text {bis }}{ }^{1}=$ índice de discriminación para actitud hacia el ejercicio físico y el deporte; $r_{\text {bis }}{ }^{2}=$ índice de discriminación para pasión por el deporte; $r_{\mathrm{bis}}{ }^{3}=$ índice de discriminación para disposición; $r_{\mathrm{bis}}{ }^{\mathrm{T}}=$ índice de discriminación de la escala total.

Los resultados reflejaron que existe una correlación moderada baja tanto entre la actitud hacia el ejercicio físico y el bienestar psicológico como entre la actitud hacia el ejercicio físico y la satisfacción con la vida. Estos resultados respaldan la información provista por Pollock et al. (1998) y por Jiménez et al. (2008), quienes mencionan una correlación significativa entre el ejercicio físico y determinados efectos saludables, como el bienestar y la satisfacción con la vida.

\section{Análisis de comparación}

Se realizó un análisis de comparación de medias mediante prueba $t$ para muestras independientes con el propósito de evaluar si existen diferencias estadísticamente significativas entre las medias de actitud hacia el ejercicio físico y el deporte de hombres y mujeres (véase Tabla 5). Los análisis reflejaron que los hombres tienen una actitud más positiva hacia el ejercicio físico y el deporte que las mujeres. También se realizó una prueba $t$ para evaluar si existen diferencias estadísticamente significativas entre las medias de bienestar psicológico y de satisfacción con la vida de las personas que practican ejercicio físico tres veces o más por semana y las medias de las que no. Los resultados revelaron que no existen diferencias significativas entre estos dos grupos.

\section{Discusión}

El presente estudio tuvo como objetivo prin- 
Tabla 4

Correlaciones entre las variables, sus medias, desviaciones estándar y alfa de Cronbach.

\begin{tabular}{|c|c|c|c|c|c|c|c|c|c|}
\hline & $\alpha$ & $S-B$ & $M$ & $D E$ & 1 & 2 & 3 & 4 & 5 \\
\hline 1. Actitud hacia el ejercicio & .88 & .87 & 12.47 & 3.97 & - & & & & \\
\hline 2. Pasión por el deporte & .73 & .72 & 5.82 & 2.73 & $.92 *$ & - & & & \\
\hline 3. Disposición & .77 & .78 & 15.14 & 1.48 & $.71 *$ & $.46^{*}$ & - & & \\
\hline 4. AHEFD-1 & .88 & .90 & 27.78 & 6.88 & $.76^{*}$ & $.59 *$ & $.33 *$ & - & \\
\hline 5. Bienestar psicológico & .85 & .88 & 80.80 & 11.19 & $.34 *$ & $.31^{*}$ & $.22 *$ & $.29^{*}$ & - \\
\hline 6. Satisfacción con la vida & .88 & .85 & 25.96 & 6.24 & $.27 *$ & $.26^{*}$ & $.20^{*}$ & $.17 *$ & $.74 *$ \\
\hline
\end{tabular}

Nota. $*=p<.01 ; \alpha=$ alfa de Cronbach; $\mathrm{S}-\mathrm{B}=$ Spearman-Brown; $\mathrm{M}=$ medias; $\mathrm{DE}=$ desviación estándar.

cipal desarrollar y validar la Escala de Actitud Hacia el Ejercicio Físico y los Deportes (AHEFD-11) en una muestra de adultos universitarios puertorriqueños. Este es el primer instrumento desarrollado totalmente en Puerto Rico para medir la actitud hacia el ejercicio físico y los deportes desde una perspectiva holística y contextualizada que toma en consideración la historia y la cultura de los puertorriqueños. Además, este estudio pretendía conocer la relación que existe entre la actitud hacia el ejercicio físico y los deportes, y entre la satisfacción con la vida y el bienestar psicológico. Esto representa un importante aporte para el desarrollo de nuevas investigaciones sobre la actitud hacia el ejercicio físico y los deportes en Puerto Rico. A su vez, permitirá análisis más amplios sobre las diferencias entre aspectos, tales como la actitud hacia la actividad física (practicada o no), la satisfacción con la vida, el bienestar psicológico y el impacto de estos en la salud física y psicológica de los puertorriqueños.

Los fundamentos teóricos y prácticos de Dosil-Díaz (2002) permitieron desarrollar 13 reactivos que se agruparon en tres subescalas: actitud hacia el ejercicio, pasión por el deporte y disposición. Estas dimensiones integran las actitudes, la pasión y las prácticas referentes a la actividad física y los deportes que puede manifestar un indi-

Tabla 5

Resultados de la prueba $t$ para el análisis de comparación de medias y tamaño del efecto.

\begin{tabular}{|c|c|c|c|c|c|c|c|}
\hline Variables & $\mathbf{n}$ & $\mathbf{M}$ & DE & $t$ & $d f$ & $p$ & $d$ \\
\hline \multicolumn{8}{|c|}{ Actitud hacia el ejercicio } \\
\hline Femenino & 203 & 27.36 & 6.72 & -2.20 & 252 & .03 & .34 \\
\hline Masculino & 51 & 29.71 & 7.12 & & & & \\
\hline \multicolumn{8}{|l|}{ Bienestar psicológico } \\
\hline Tres veces por semana & 108 & 83.03 & 10.09 & 1.36 & 174 & .18 & .21 \\
\hline Menos de tres veces & 68 & 80.87 & 10.53 & & & & \\
\hline Valores perdidos & 79 & & & & & & \\
\hline \multicolumn{8}{|l|}{ Satisfacción con la vida } \\
\hline Femenino & 108 & 26.71 & 6.45 & 0.29 & 174 & .27 & .06 \\
\hline Masculino & 68 & 26.34 & 5.20 & & & & \\
\hline Valores perdidos & 79 & & & & & & \\
\hline
\end{tabular}

Nota. $\mathrm{n}=$ participantes; $\mathrm{M}=$ medias; $d f=$ grados de libertad; $\mathrm{DE}=$ desviación estándar; $t=$ valor de la prueba $t ; p=$ significancia; $d=$ tamaño del efecto $d$ de Cohen. $(\mathrm{N}=255)$. 
viduo. El análisis factorial exploratorio confirmó la presencia de estas tres dimensiones. En cuanto a las subescalas del instrumento, la dimensión denominada actitud hacia el ejercicio corresponde a la actitud de las personas hacia el ejercicio físico según sus prioridades, consideraciones, tiempo y dedicación (Pollock et al., 1998). Estas características son importantes para el resultado de una actividad física adecuada.

La segunda dimensión, pasión por el deporte, está asociada a los factores relacionales de la pasión deportiva que experimentan las personas por algún deporte en particular. Se trata de asistir, observar y modificar el estilo de vida para participar en algún evento deportivo, sea de manera presencial o como espectador a través de los diversos medios de comunicación. Según Dosil-Díaz (2002), existen elementos comportamentales y afectivos que describen esta dimensión y distinguen algunas características del apasionado por el deporte.

La tercera dimensión, disposición, está relacionada con los factores de disponibilidad de la persona para realizar algún ejercicio físico. Gucciardi et al. (2009) explican que, en el ámbito de la psicología de la actividad física y el deporte, se emplea un concepto llamado fortaleza mental, definido como "una colección de valores, actitudes, emociones y cogniciones que influyen en la manera como una persona accede, responde y evalúa eventos de alto nivel de exigencia para lograr sus metas de manera consistente".

A estos efectos, la predisposición de la persona para realizar actividad física y deportiva puede ser una característica de la manera como el individuo evalúa eventos de alto nivel de exigencia. En cuanto a los hallazgos sobre la actitud hacia el ejercicio físico, el bienestar psicológico y la satisfacción con la vida, todos los coeficientes $r$ de Pearson obtenidos en los análisis fueron estadísticamente significativos. Los resultados re- flejaron que existe una correlación moderada baja tanto entre la actitud hacia el ejercicio físico y el bienestar psicológico como entre la actitud hacia el ejercicio físico y la satisfacción con la vida. Estos resultados concuerdan con De Miguel-Calvo, Schweiger-Gallo, de las Mozas-Majano y Hernández-López (2011) que comentan que realizar ejercicio físico de forma sistemática y controlada tiene una influencia positiva en la productividad y el bienestar de las personas. Del mismo modo, estos resultados también concuerdan con Jiménez-Moral, Zagalaz-Sánchez, Molero, Pulido-Martos y Ruiz (2013), quienes argumentan que existe una asociación positiva entre el ejercicio físico, la felicidad subjetiva y la satisfacción con la vida. En consecuencia, estos resultados contribuyen a entender más la importancia que tiene el ejercicio físico para las personas como práctica dirigida a mejorar su calidad de vida.

Por otro lado, se realizó un análisis de comparación de medias mediante prueba $t$ con el propósito de evaluar la existencia de diferencias estadísticamente significativas entre las medias de actitud hacia el ejercicio físico y el deporte de hombres y mujeres. Se encontró que los hombres poseen una mejor actitud hacia el ejercicio físico que las mujeres. Estos resultados van alineados con Cossío-Bolaños et al. (2017), quien encontró en su estudio Patrones de actividad fisica de adolescentes escolares: Validez, confiabilidad y propuesta de percentiles para su evaluación que los hombres presentan mayores niveles de actitud física que las mujeres. También, Sevil-Serrano, Práxedes-Pizarro, Zaragoza-Casterad, del Villar-Álvarez y García-González (2017) en su estudio Barreras percibidas para la práctica de actividad física en estudiantes universitarios: Diferencias por género y niveles de actividad física mencionan que, atendiendo al género, los hombres obtienen mayor cumplimiento de práctica de actividad física (76.7\%) y mayores niveles de 
actividad física moderada y vigorosa semanales (384.93) que las mujeres. Esto puede explicar las diferencias significativas que existen entre hombres y mujeres en cuanto a la actividad física.

Como menciona la WHO (2010), en el año 2010 a nivel mundial, alrededor del $23 \%$ de los adultos mayores de 18 años no se mantenía suficientemente activo (un 20\% de los hombres y un $27 \%$ de las mujeres). En los países de ingresos altos, el $26 \%$ de los hombres y el $35 \%$ de las mujeres no hacía suficiente ejercicio físico, frente a un $12 \%$ de los hombres y un $24 \%$ de las mujeres en los países de ingresos bajos. En consecuencia, se percibe un patrón de diferencias entre géneros en cuanto al ejercicio físico. Los roles creados por el entorno sociocultural a través del tiempo han generado una serie de conductas, valores y tareas diferenciadas por género. Las tareas menos onerosas estarían reservadas a la mujer y al hombre, todas las tareas físicas (Carrillo-Montoya, Tereso-Ramírez, \& González-Ramírez, 2018). El contexto también define el tipo de actividad física que se lleva a cabo, por ejemplo, deportes, juego, educación, trabajo y ejercicio, entre otros. Estas distintas actividades están fuertemente influenciadas por la cultura (Latorre-Román et al., 2017). Esto puede explicar la causa por la que el hombre manifiesta, a lo largo del tiempo, una actitud más positiva hacia el ejercicio físico y los deportes que las mujeres.

Estos resultados no guardan relación con el trabajo de Jiménez et al. (2008) quienes mencionan que la prescripción de ejercicio físico va encaminada habitualmente a lograr determinados efectos saludables en relación con diferentes parámetros (frecuencia, duración intensidad, tipo de ejercicio, etc.). En el estudio en cuestión, las personas que realizan ejercicio físico dos días o menos a la semana no lo hacen de acuerdo con la frecuencia, duración e intensidad recomendadas por la WHO (2018). Sin embargo, en los resultados obtenidos en esta investigación, los participantes no presentaron diferencias significativas en cuanto a su bienestar psicológico y satisfacción con la vida. Por lo tanto, estos resultados no están en la misma línea de otras investigaciones.

Tomando esto en consideración, dentro de la gran cantidad de estudios dirigidos a conocer los beneficios del ejercicio físico, se ha demostrado que el ejercicio físico tiene un efecto beneficioso para la salud (Barbany, 2015; Jiménez-Moral et al., 2013). Por otra parte, otros autores argumentan que, pese a los beneficios conocidos sobre la actividad física, un 30\% de la población mundial no alcanza los niveles de actividad física recomendados (Franco et al., 2015). En cuanto a los efectos de bienestar psicológico y satisfacción con la vida, las personas que realizan actividad física con mayor frecuencia muestran efectos negativos menores en la salud y el bienestar psicológico (Haith-Cooper et al., 2018). Por el contrario, en el presente estudio, quienes realizan ejercicio físico dos veces o menos por semana no mostraron diferencias significativas con respecto al bienestar psicológico y la satisfacción con la vida. Franco et al. (2015) pueden brindar una posible explicación de este fenómeno. Franco menciona que, a pesar de que las personas manifiestan tener alguna idea sobre los beneficios del ejercicio y los deportes, solo un 30\% practica alguna actividad física. Sin embargo, entre las causas de este bajo porcentaje se encuentran la baja conciencia de los beneficios que puede traer la actividad física y el escaso acceso a las condiciones ambientales y económicas que permiten su práctica. Esto, puede explicar por qué no hubo diferencias significativas entre las personas que llevaban a cabo ejercicios físicos tres veces o más por semana y las que no con respecto a sus niveles de satisfacción con la vida y bienestar psicológico.

En síntesis, las propiedades psicométricas obtenidas por la AHEFD-11 demuestran ín- 
dices de confiabilidad, de consistencia interna y de validez de acuerdo con lo esperado. Esto fue verificado al observar la consistencia interna del instrumento final. De igual forma, la validez de construcción lógica del instrumento fue examinada a través de un análisis factorial exploratorio. Los análisis psicométricos de la AHEFD-11 demuestran que el instrumento es apropiado. Sin embargo, al ser un instrumento desarrollado en un estudio exploratorio, se lo debe poner a prueba utilizando una muestra mayor. Finalmente, esta escala podrá ser utilizada como una herramienta útil para aquellos profesionales que deseen auscultar las actitudes de las personas hacia el ejercicio físico y los deportes. Del mismo modo, detectar a individuos que necesiten psicoeducación en este aspecto, utilizando la escala como una herramienta para la prevención de enfermedades por falta de actividad física $y$, a su vez, para la promoción del ejercicio físico y los deportes.

\section{Limitaciones}

El tipo de muestreo utilizado fue por disponibilidad, lo que conlleva una reducida generalización de los resultados, que son relevantes sólo para los participantes del estudio. Por otra parte, dado el uso de plataformas electrónicas, se puede esperar que la muestra sea más representativa de un sector poblacional (usuarios frecuentes de tecnología). Por último, no se pudo examinar la confiabilidad de la escala a través del tiempo y sólo se examinó la confiabilidad de las escalas a través de sus elementos.

\section{Recomendaciones}

Recomendamos administrar la AHEFD-11 a otra muestra de participantes para realizar el proceso de validación cruzada, así como para evaluar la validez convergente y divergente del instrumento final. Por último, recomendamos la validación de la AHEFD-11 en otras poblaciones hispanoamericanas para auscultar sus propiedades psicométricas.

\section{Conclusión}

La construcción y validación de la Escala de Actitud Hacia el Ejercicio Físico y los Deportes aportan al quehacer psicológico en la isla y al desarrollo de nuevos conocimientos sobre la actitud del puertorriqueño hacia la actividad física y los deportes. En primer lugar, la AHEFD-11 tiene la capacidad de contribuir a investigaciones científicas en el contexto sociocultural puertorriqueño que permitan entender con mayor profundidad la actitud de las personas hacia la actividad física y los deportes, tanto positiva como negativa, y su repercusión en la salud física y mental de la población. Por otra parte, en el ambiente clínico, la AHEFD-11 contribuye como herramienta práctica que los psicólogos pueden incorporar para tratar temas relacionados con las actitudes hacia el ejercicio, la actividad física, la salud física y los deportes como medio de distracción, entretenimiento y estilo de vida. Las tres subescalas del instrumento permiten al terapeuta identificar la actitud del cliente hacia el ejercicio o la participación en algún evento deportivo como método de distracción. No cabe duda de que estos dos aportes incrementan el valor de este instrumento en Puerto Rico.

\section{Referencias}

American College of Sports Medicine. (2000). ACSMs guidelines for exercise testing and prescription 
(6 $6^{\text {e }}$ ed.). Philadelphia, PA: Lippincott Williams \& Wilkins.

Barbany, J. R. (2015). Fisiología del ejercicio físico y del entrenamiento. Barcelona, España: Paidotribo.

Carrillo-Montoya, T. J., Tereso-Ramírez, L., \& González-Ramírez, E. (2018). La familia y la construcción del posicionamiento laboral en las mujeres. Un análisis desde el Trabajo Social. Prospectiva, Revista de Trabajo Social e Intervención Social, 25, 91111. doi: 10.25100/prts.v0i25.5802

Cossío-Bolaños, M., Méndez-Cornejo, J., Luarte-Rocha, C., Vargas-Vitoria, R., Canqui-Flores, B., \& Gomez-Campos, R. (2017). Patrones de actividad física de adolescentes escolares: Validez, confiabilidad y propuesta de percentiles para su evaluación. Revista Chilena de Pediatría, 88(1), 73-82. doi: 10.1016/j. rchipe.2016.07.010

De Miguel-Calvo, J. M., Schweiger-Gallo, I., de las Mozas-Majano, O., \& Hernández-López, J. M. (2011). Efecto del ejercicio físico en la productividad laboral y el bienestar. Revista de Psicología del Deporte, 20(2), 589-604. Recuperado de https://www.rpd-online.com

Department of Health, Physical Activity, Health Improvement and Protection. (2011). Start Active, Stay Active: A report on physical activity for health from the four home countries' chief medical officers. Recuperado de https://www.gov.uk

Diener, E., Emmons, R. A., Larsen, R. J., \& Griffin, S. (1985). The Satisfaction With Life Scale. Journal of Personality Assessment, 49(1), 71-75. doi: 10.1207/ s15327752jpa4901_13

Dosil-Díaz, J. (2002). Escala de Actitudes hacia la Actividad Física y el Deporte (E.A.F.D). Cuadernos de Psicología del Deporte, 2(2), 43-55. Recuperado de https://revistas.um.es/cpd/index

Field, A. P. (2013). Discovering statistics using SPSS (4 ${ }^{\mathrm{a}}$ ed.). Londres, Inglaterra: SAGE.

Franco, M. R., Tong, A., Howard, K., Sherrington, C., Ferreira, P. H., Pinto, R. Z., \& Ferreira, M. L. (2015). Older people's perspectives on participation in physical activity: A systematic review and thematic synthesis of qualitative literature. British Journal of Sports Medicine, 49(19), 1268-1276. doi: 10.1136/ bjsports-2014-094015

González-Rivera, J. A., Quintero-Jiménez, N., Veray-Alicea, J., \& Rosario-Rodríguez, A. (2016). Adaptación y validación de la Escala de Bienestar Psicológico de Ryff en una muestra de adultos puertorriqueños. Salud y Conducta Humana, 3(1), 1-14. Recuperado de http://rsych.com

Gucciardi, D. F., Gordon, S., \& Dimmock, J. A. (2009). Advancing mental toughness research and theory using personal construct psychology. International Review of Sport and Exercise Psychology, 2(1), 5472. doi: 10.1080/17509840802705938

Haith-Cooper, M., Waskett, C., Montague, J., \& Horne, M. (2018). Exercise and physical activity in asylum seekers in Northern England: Using the theoretical domains framework to identify barriers and facilitators. BMC Public Health, 18(1), 1-11. doi: 10.1186/ s12889-018-5692-2

Hassmén, P., Koivula, N., \& Uutela, A. (2000). Physical exercise and psychological well-being: A population study in Finland. Preventive Medicine, 30(1), 17-25. doi: 10.1006/pmed.1999.0597

Hatcher, L. (1994). A step-by-step approach to using the SAS System for Factor Analysis and Structural Equation Modeling. Cary, NC: SAS Institute.

IBM Corporation. (2016). IBM SPSS Statistics for Windows, Version 24.0 [Software de cómputo]. Armonk, NY: IBM.

Jiménez, M. G., Martínez, P., Miró, E., \& Sánchez, A. I. (2008). Bienestar psicológico y hábitos saludables: ¿Están asociados a la práctica de ejercicio físico? International Journal of Clinical Health \& Psychology, 8(1), 185-202. Recuperado de http://www.aepc. es/ijchp/busca.php?coid=English?

Jiménez-Moral, J. A., Zagalaz-Sánchez, M. L., Molero, D., Pulido-Martos, M., \& Ruiz, J. R. (2013). Capacidad aeróbica, felicidad y satisfacción con la vida en adolescentes españoles. Revista de Psicología del De- 
porte, 22(2), 429-436. Recuperado de https://www. rpd-online.com

Kenyon, G. S. (1968). Six scales for assessing attitude toward physical activity. Research Quarterly. American Association for Health, Physical Education and Recreation, 39(3), 566-574. doi: 10.1080/10671188.1968.10616581

Latorre-Román, P. A., Moreno del Castillo, R., Lucena-Zurita, M., Salas-Sánchez, J., García-Pinillos, F., \& Mora-López, D. (2017). Physical fitness in preschool children: Association with sex, age and weight status. Child: Care, Health and Development, 43(2), 267-273. doi: 10.1111/cch.12404

Malina, R. M., \& Katzmarzyk, P. T. (2006). Physical activity and fitness in an international growth standard for preadolescent and adolescent children. Food and Nutrition Bulletin, 27(4), 295-313. doi: 10.1177/15648265060274S511

Meseguer-Zafra, M., Rosa-Guillamón, A., García-Cantó, E. , Rodríguez-García, P., Pérez-Soto, J. J. , Tárraga-López, P. J., ...Tárraga-Marcos, M. (2019). Influence of a programme of therapeutic exercise on different clinical indicators related to dyslipidaemia in adult subjects aged between 26 to 73 years with a cardiovascular risk factor. Hipertensión $y$ Riesgo Vascular, 36(1), 21-27. doi: 10.1016/j.hipert.2018.02.001

Montero, I., \& Leon, O. G. (2007). A guide for naming studies in psychology. International Journal of Clinical and Health Psychology, 7(3), 847-862. Recuperado de http://www.aepc.es/ijchp/busca.php?coid=English?

Pedišić, Ž., Dumuid, D., \& Olds, T. S. (2017). Integrating sleep, sedentary behaviour, and physical activity research in the emerging field of time-use epidemiology: Definitions, concepts, statistical methods, theoretical framework, and future directions. Kinesiology, 49(2), 252-269. Recuperado de https://hrcak.srce.hr/ojs/index.php/kinesiology/index

Pollock, M. L., Gaesser, G. A., Butcher, J. D., Després, J. P., Dishman, R. K., Franklin, B. A, \& Garber, C.
E. (1998). ACSM position stand: The recommended quantity and quality of exercise for developing and maintaining cardiorespiratory and muscular fitness, and flexibility in healthy adults. Medicine \& Science in Sports \& Exercise, 30(6), 975-991. doi: 10.1249/00005768-199806000-00032

PsychData. (s. f.). Create online surveys with confidence and ease [Herramienta en línea]. Recuperado de https://www.psychdata.com

Rebolledo-Cobos, R. C., Teixeira, B., \& Correa, C. (2014). Asma, estrés oxidativo y ejercicio físico: Revisión de la literatura. Fisioterapia, 36(1), 40-48. doi: 10.1016/j.ft.2013.06.002

Ryff, C. D. (1989). Happiness is everything, or is it? Explorations on the meaning of psychological well-being. Journal of Personality and Social Psychology, 57(6), 1069-1081. doi: 10.1037/0022-3514.57.6.1069

Sevil-Serrano, J., Práxedes-Pizarro, A., Zaragoza-Casterad, J., del Villar-Álvarez, F., \& García-González, L. (2017). Barreras percibidas para la práctica de actividad física en estudiantes universitarios: Diferencias por género y niveles de actividad física. Universitas Psychologica, 16(4), 1-15. doi: 10.11144/ Javeriana.upsy 16-4.bppa

Stevens, J. (2002). Applied multivariate statistics for the social sciences ( $4^{\mathrm{a}}$ ed.). Mahwah, NJ: Lawrence Erlbaum.

Taylor, H. L., Buskirk, E., \& Henschel, A. (1955). Maximal oxygen intake as an objective measure of cardio-respiratory performance. Journal of Applied Physiology, 8(1), 73-80. doi: 10.1152/jappl.1955.8.1.73

World Health Organization. (2010). Global recommendations on physical activity for health. Suiza: World Health Organization. Recuperado de https://www. who.int

World Health Organization. (2018). Benefits of physical activity and risks of an insufficient level of physical activity. Recuperado de https://www.who.int 